\title{
Methicillin-resistant Staphylococcus aureus in Metabolic Syndrome Patients at the Mbouda Hospitals, West Region of Cameroon
}

\author{
Wiliane Jean Takougoum Marbou ${ }^{1}$, Victor Kuete ${ }^{2}$ \\ 1. Biochemistry, Faculty of Sciences, University of Dschang, Dschang, CMR 2. Biochemistry, University of Dschang, \\ Dschang, CMR
}

Corresponding author: Victor Kuete, kuetevictor@yahoo.fr

\section{Abstract \\ Background}

Studies have revealed an increased risk of contracting Staphylococcus aureus infections in patients suffering from metabolic diseases. Methicillin-resistant Staphylococcus aureus (MRSA) in metabolic syndrome subjects is less reported in the medical literature. This study aimed at isolating and establishing the distribution of antibiotic-resistant Staphylococcus aureus from faecal samples in metabolic syndrome subjects from Mbouda Hospitals, West Region of Cameroon.

\section{Methods}

A cross-sectional study was conducted from May 2016 to May 2018 in 114 participants in whom Staphylococcus aureus was detected. Thirty (30) participants were suffering from metabolic syndrome and 84 did not suffer from this pathology. Staphylococcus aureus isolation was based on culture and confirmed by polymerase chain reaction (PCR) of the nuc gene. The Kirby-Bauer disk diffusion method was used for drug susceptibility assay. Molecular detection of the mecA gene by PCR was performed to screen MRSA.

\section{Results}

From the 114 Staphylococcus aureus isolates, the prevalence of the mecA gene confirming MRSA was $79.82 \%$, higher than that of methicillin-sensitive Staphylococcus aureus (MSSA) (20.17\%). The frequency of MRSA was higher in participants with metabolic syndrome (80.00\%) compared to non-metabolic syndrome (79.76\%) participants without significant difference $(\mathrm{p}=0.977)$. The antimicrobial susceptibility test revealed that the amikacin susceptibility profile was significantly different in metabolic and non-metabolic syndrome participants $(\mathrm{p}=0.037$, chi-square $=6.59$ ). Regarding metabolic syndrome status, $72.62 \%$ of isolates were multidrug-resistant in non-metabolic syndrome participants versus $63.33 \%$ in metabolic syndrome participants.

Received 02/28/2020

Review began 03/02/2020 Review ended 03/11/2020 Published 03/15/2020

๑) Copyright 2020

Marbou et al. This is an open access article distributed under the terms of the Creative Commons Attribution License CC-BY 4.0., which permits unrestricted use, distribution, and reproduction in any medium, provided the original author and source are credited.

\section{Conclusion}

This study suggests that metabolic syndrome patients harbour MRSA strains in their intestines even as the difference was not statistically significant with non-metabolic syndrome participants. The need for appropriate antimicrobial use to halt or at least limit the spread of resistance is suggested in the care of metabolic syndrome patients and the entire population.

Categories: Infectious Disease, Epidemiology/Public Health

Keywords: staphylococcus aureus, intestinal carriage, oxacillin resistance, metabolic syndrome, mbouda-cameroon

\section{Introduction}

Some studies have revealed that there is an increased risk of contracting Staphylococcus aureus infections in patients with diabetes and obesity than in those without [1]. Obesity and type 2 diabetes are recognized as a determining factor in the development of metabolic syndrome [2]. Methicillin-resistant Staphylococcus aureus epidemiology in the metabolic syndrome patients is less known. Staphylococcus aureus is an important opportunistic pathogen responsible for a variety of diseases. It is a gram-positive bacterium that occurs naturally in and on the human body. Staphylococcus aureus is found in the axillae (8\%), chest/abdomen (15\%), perineum (22\%), and intestine (17-31\%) [3]. In healthy individuals, an intestinal carriage of $20 \%$ for Staphylococcus aureus has been reported and although nasal carriage may predispose them to intestinal carriage, sole intestinal carriage was also detected [4]. From the nasopharynx, the bacterium is propagated on the skin by aerosol and is often present on clothes and dander [4]. As staphylococci are resistant to desiccation, transmission can not only be direct, through the hands of health care workers in hospitals but also indirectly by objects and dust [5]. These several niches in the body form major reservoirs for the Staphylococcus aureus infection. Staphylococcus aureus colonisation is a major risk factor for staphylococcal infections. 
The human intestinal tract harbours a large number of cultivable and non-cultivable bacteria. The colonization of the gastrointestinal tract by Staphylococcus aureus has been documented as potential sources of both endogenous and exogenous staphylococcal infections [6-7]. Stools specimens can surely be significant as a source of environmental contamination and have been identified as a possible source of antibiotic-resistant Staphylococcus aureus especially methicillin-resistant Staphylococcus aureus (MRSA).

MRSA is becoming more of a public health concern. MRSA causes skin infections, such as paronychia, styles, and furunculosis and life-threatening systemic infections such as sepsis, pneumonia, and endocarditis [8]. The resistance of Staphylococcus aureus to methicillin is caused by a gene name mecA, which alters the site at which methicillin binds to kill the organism. Staphylococcus aureus bacteria resistant to methicillin are also resistant to many other antibiotics because the same cellular process is used by bacteria to be resistant to other antibiotics [9].

To the very best of our knowledge, very few studies have focused on the intestinal reservoir of MRSA. Similarly, no comprehensive studies regarding the distribution of antibiotic-resistant Staphylococcus aureus from faecal samples in metabolic syndrome subjects have been reported in the medical literature. In this study, we presumed that the faeces of metabolic syndrome subjects might contain MRSA strains and would have the possibility to serve as a potential source of MRSA dissemination in the Bamboutos Division, West Region of Cameroon. The aim of this study, therefore, was to determine the distribution of the antibiotic-resistant Staphylococcus aureus from faecal samples in metabolic syndrome subjects from Mbouda Hospitals, West Region of Cameroon.

\section{Materials And Methods \\ Subjects and study design}

This study was conducted in a sub-sample of the study carried out to estimate the prevalence of metabolic syndrome and its components in Bamboutos Division's adults (20 years old and older), West Region of Cameroon [10]. One hundred and fourteen (114) patients from whom the presence of Staphylococcus aureus was detected in the faeces were included in this study. Four-hundred ninety (490) were excluded. This was a cross-sectional study conducted from May 2016 to May 2018. Patients coming for consultation for enteric disorders at the Mbouda AD LUCEM and District Hospitals, two reference hospitals in Bamboutos Division, were recruited. Human immunodeficiency virus (HIV) positive patients, pregnant women, patients under antibiotic treatment, and participants with positive serology for Hepatitis B and C were not included in this study.

\section{Data collection}

Data collection and examination was performed as part of the study previously described by Marbou and Kuete (2019) [10]. Briefly, they comprised standardized questionnaire, clinical examinations with blood pressure at rest (OMROM 705), fasting blood glucose (ACCU-CHEK, Mannheim, Germany), lipid profile (MaestroNano, MaestroGen, USA), waist circumference measurement and stool collection. Metabolic syndrome was defined using the Joint Interim Statement of the International Diabetes Federation Task Force on Epidemiology and Prevention definition [11].

\section{Stools collection}

Six hundred and four (604) stool specimens were collected using sterile bottles (Viamed, Miami Lakes, Florida). The specimens collected were first inoculated in nutrient agar (NA) (HiMedia, Mumbai, India), subcultured in mannitol salt agar (MSA) (Uptima/Interchim, France), and aerobically incubated for 24 hours at $37^{\circ} \mathrm{C}$. Bacterial colonies showing the typical characteristics of Staphylococcus aureus, including goldenyellow colour colonies on MSA, were subjected to molecular confirmation by polymerase chain reaction (PCR).

\section{Molecular confirmation of Staphylococcus aureus}

In this study, the TENT (Tris-EDTANaCl-TritonX100) method for Staphylococcus aureus deoxyribonucleic acid (DNA) extraction was used as described by Hassanzadeh et al. (2016) [12]. Staphylococcus aureus was confirmed by PCR of the nuc gene as previously described by Brakstad et al. (1992) using primers listed in Table 1 [13]. 


\section{Cureus}

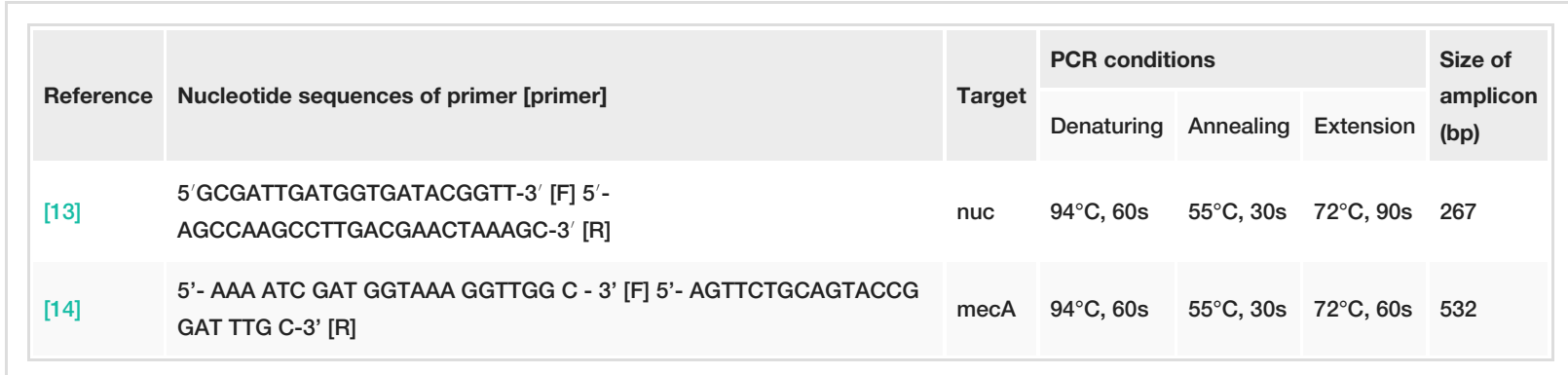

TABLE 1: Polymerase chain reaction (PCR) primers and conditions used in this study

[F]: Forward primer; [R]: Reverse primer; PCR: Polymerase chain reaction

\section{Antimicrobial susceptibility testing}

The Kirby-Bauer disk diffusion method was performed as per the recommendations of the Clinical and Laboratory Standards Institute (CLSI) guidelines [14]. A bacterial suspension equivalent to the $0.5 \mathrm{McFarland}$ turbidity standard was prepared and inoculated on Mueller-Hinton agar (MHA) (Accumix, Mol, Belgium) supplemented with $2 \% \mathrm{NaCl}$. Standard antimicrobial disks representing multiple drug classes were subsequently set for oxacillin (beta-lactam) $(1 \mu \mathrm{g})$, gentamicin (aminoglycosides) $(10 \mu \mathrm{g})$, amikacin (aminoglycosides) $(30 \mu \mathrm{g})$, chloramphenicol (chloramphenicol) $(30 \mu \mathrm{g})$, doxycycline (tetracycline) $(30 \mu \mathrm{g})$, and co-trimoxazole (sulfonamides) $(23.75 / 1.25 \mu \mathrm{g})$ (Becton Dickinson and Company, Sparks, Maryland). The plates were incubated at $37^{\circ} \mathrm{C}$ for 24 hours. An inhibition zone diameter of each antimicrobial was then measured and interpreted as resistant (R), intermediate (I), and sensitive (S). mecA-negative Staphylococcus aureus ATCC 29213 and mecA-positive Staphylococcus aureus ATCC 33591 were used as the control in each test run. Inhibition zones were interpreted according to CLSI guidelines [14].

\section{Molecular detection of the mecA gene by PCR}

The presence of the mecA gene responsible for the resistance of Staphylococcus aureus to methicillin was detected by PCR in all confirmed Staphylococcus aureus resistant to Oxacillin. mecA was amplified using specifics mecA primers (Table 1) as described by Kumurya, Gwarzo, and Uba (2015) [15]. Amplification was done in a PCR solution of $20 \mu \mathrm{l}$ containing $11.25 \mu \mathrm{l}$ nuclease-free water, $2.5 \mu \mathrm{l}$ of 10xPRC buffer (New England Biolab, Hitchin, UK), $2.0 \mu \mathrm{l}$ dNTP Mix (Thermo Fischer Scientific, UK), $1 \mu \mathrm{l}$ of forward and reverse primer each, $2 \mu \mathrm{l}$ of DNA template, and $0.25 \mu \mathrm{l}$ of Taq DNA polymerase (New England Biolab, Hitchin, UK). DNA amplification was carried out in a Techne PCR system TC-5000 thermocycler (Bibby Scientific Ltd., Essex, UK) with the following thermal-cycling profile: an initial denaturation step at $94^{\circ} \mathrm{C}$ for five min, followed by one cycle of amplification starting by denaturation at $94^{\circ} \mathrm{C}$ for $30 \mathrm{~s}$, annealing at $55^{\circ} \mathrm{C}$ for $30 \mathrm{~s}$, and extension at $72^{\circ} \mathrm{C}$ for $60 \mathrm{~s}$, ending with a final extension step at $72^{\circ} \mathrm{C}$ for five min. mecA-positive Staphylococcus aureus ATCC 33591 was used and PCR products were visualized after electrophoresis on 2\% agarose gel stained with ethidium bromide.

\section{Ethical consideration}

Participants received oral and written information about the study and signed an informed consent form. The protocol was approved by the Cameroon National Ethics Committee (CNEC), Ministry of Public Health (reference number, 2018/06/

1054/CE/CNERSH). The study was conducted in agreement with the Declaration of Helsinki.

\section{Statistical analysis}

The data collected were analyzed by Epi Info ${ }^{\mathrm{TM}}$ version 7.2.2.6 (CDC, 1600 Clifton Road, Atlanta). The chisquare $(\chi 2)$ test was used to evaluate the relationship between antimicrobial resistance and specific variables. A p-value of $<0.05$ was considered statistically significant.

\section{Results}

A total of 114 participants from whom the Staphylococcus aureus was isolated, and confirmed by PCR (using the nuc gene) (Figure 1), in the faeces were studied. Thirty (30) participants were suffering for metabolic and 84 did not suffer from this pathology. The presence of the mecA gene responsible for the resistance of Staphylococcus aureus to methicillin was detected by PCR in all confirmed Staphylococcus aureus (Figure 2). The prevalence of the mecA gene confirming MRSA was $79.82 \%$ ( $n=91$ ). 


\section{Cureus}

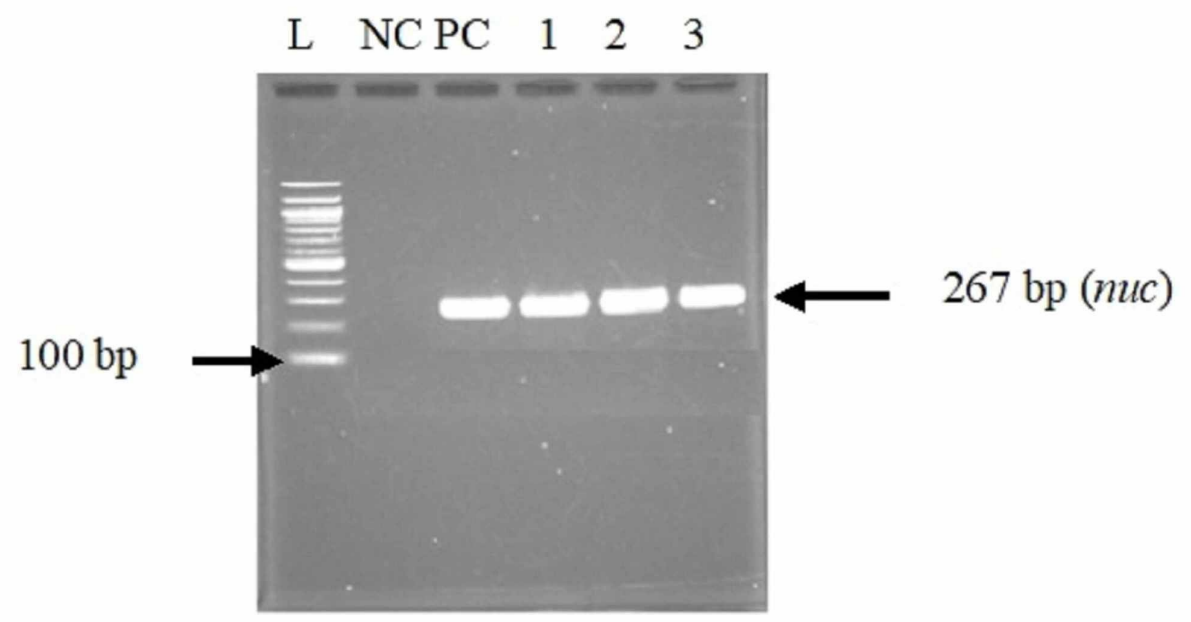

FIGURE 1: Gel electrophoresis profile of the nuc gene for the confirmation of Staphylococcus aureus isolates

Lane L: molecular size marker (100 bp DNA ladder); Lane NC: negative control; Lane PC: positive control; Lanes 1, 2, and 3: positive Staphylococcus aureus

DNA: deoxyribonucleic acid

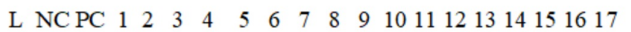

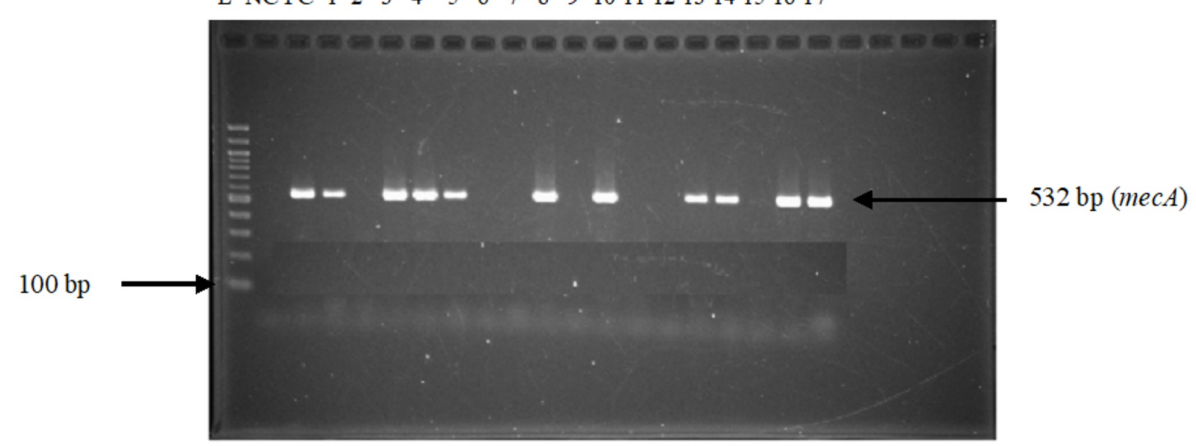

FIGURE 2: Gel electrophoresis profile of the mecA gene for the detection of methicillin-resistant Staphylococcus aureus

Lane L: molecular size marker (100 bp DNA ladder); Lane NC: negative control; Lane PC: positive control; Lanes 1, 3, 4, 5, 8, 10, 13, 14, 16, and 17: methicillin-resistant Staphylococcus aureus; Lanes 2, 6, 7, 11, 12 and 15: methicillin-sensitive Staphylococcus aureus

DNA: deoxyribonucleic acid

The age range of the study participants was from 20-83 years. The frequency of MRSA was higher in participants with metabolic syndrome (80.00\%) as compared to non-metabolic syndrome $(79.76 \%)$ participants without a significant difference $(\mathrm{p}=0.977)$ (Figure 3). 


\section{Cureus}

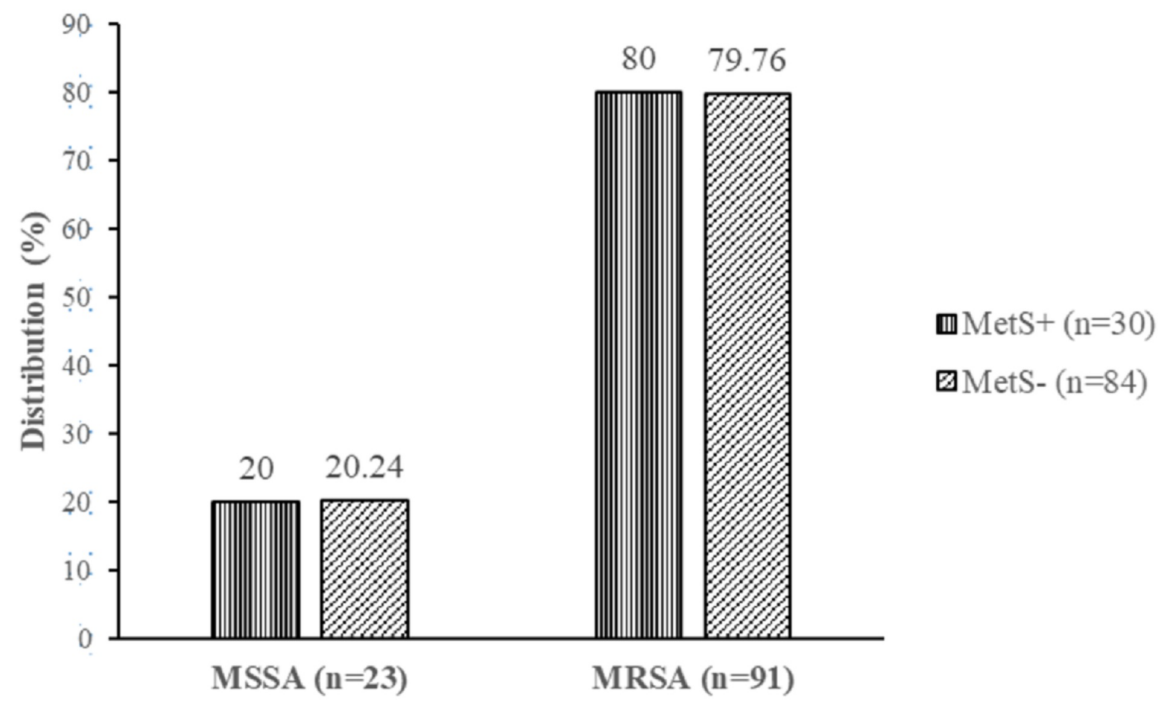

\section{FIGURE 3: Distribution of methicillin-sensitive and resistant Staphylococcus aureus according to metabolic syndrome status}

MSSA: methicillin-sensitive Staphylococcus aureus; MRSA: methicillin-resistant Staphylococcus aureus; MetS: metabolic syndrome

The distribution of Staphylococcus aureus among participants with regard to gender and age was studied. Most of the Staphylococcus aureus were isolated in the age groups of 20 to <30 years. In the 114 Staphylococcus aureus isolates, the frequency of MRSA 91 (79.83\%) was higher than that of MSSA 23 (20.17\%). The frequency of MRSA in males was 41 (80.39\%) and that of females was 50 (79.37\%). A high frequency of MRSA was observed in participants aged 60 to $<70$ and $\geqslant 70$ years $(92.86 \%$ and 100.00 ), respectively, in which a lower incidence of MSSA was observed (7.14\% and $0.00 \%$ ), respectively (Table 2).

\begin{tabular}{|c|c|c|c|c|}
\hline \multirow{2}{*}{ Variables } & & \multicolumn{2}{|c|}{ Presence of Staphylococcus aureus } & \multirow{2}{*}{ Total (N) } \\
\hline & & MSSA (N (\%)) & MRSA (N (\%)) & \\
\hline \multirow{2}{*}{ Sex } & Male & $10(19.61)$ & 41 (80.39) & 51 \\
\hline & Female & $13(20.63)$ & $50(79.37)$ & 63 \\
\hline \multirow{6}{*}{ Age group (year) } & $20-<30$ & $11(35.48)$ & $20(64.52)$ & 31 \\
\hline & $30-<40$ & $1(11.11)$ & $8(88.89)$ & 9 \\
\hline & $40-<50$ & $7(25.93)$ & $20(74.07)$ & 27 \\
\hline & $50-<00$ & $3(15.19)$ & $10(84.21)$ & 19 \\
\hline & $60-<70$ & $1(7.14)$ & $13(92.86)$ & 14 \\
\hline & $\geq 70$ & $0(0.00)$ & $14(100.00)$ & 14 \\
\hline
\end{tabular}

TABLE 2: Faecal carriage of Staphylococcus aureus among participants with regard to gender and age

MSSA: methicillin-sensitive Staphylococcus aureus; MRSA: methicillin-resistant Staphylococcus aureus; MetS: metabolic syndrome

The overall susceptibility profiles of Staphylococcus aureus isolates are shown in Table 3. The antimicrobial susceptibility test revealed that the resistance of Staphylococcus aureus to oxacillin was higher in participants with metabolic syndrome than non-metabolic syndrome participants (100.00\% and 92.86\%) respectively, non-significant at $\mathrm{p}=0.322$ (chi-square $=2.26$ ). Amikacin susceptibility profile was significantly different in metabolic and non-metabolic syndrome participants $(\mathrm{p}=0.037$, chi-square $=6.59)$. Eight $(9.52 \%)$, 17 (20.24\%) and 59 (70.24\%) of Staphylococcus aureus were respectively resistant, Intermediate and sensitive 


\section{Cureus}

to amikacin in metabolic syndrome versus 0 (0.00\%), 12 (40.00\%) and 18 (60.00\%) in non-metabolic syndrome participants, respectively. In non-metabolic syndrome participants, doxycycline had the highest overall resistance of $85.71 \%$, followed by co-trimoxazole (64.29\%) and chloramphenicol (51.19\%) while amikacin had the highest overall sensitivity of $70.24 \%$ followed by gentamicin (64.29\%).

\begin{tabular}{|c|c|c|c|c|}
\hline Antibiotics & Resistant, N (\%) & Intermediate, N (\%) & Sensitive, N (\%) & $X^{2}$ (probability) \\
\hline Oxacillin ${ }^{a}$ & 78 (92.86) & $2(2.38)$ & $4(4.76)$ & \multirow{2}{*}{$2.26(0.322)$} \\
\hline Oxacillin ${ }^{b}$ & $30(100.00)$ & $0(0.00)$ & $0(0.00)$ & \\
\hline Gentamicin $^{\mathrm{a}}$ & $20(23.81)$ & $10(11.90)$ & $54(64.29)$ & \multirow{2}{*}{$0.99(0.610)$} \\
\hline Gentamicin $^{b}$ & $6(20.00)$ & $2(6.67)$ & $22(73.33)$ & \\
\hline Amikacin $^{\mathrm{a}}$ & $8(9.52)$ & $17(20.24)$ & $59(70.24)$ & \multirow{2}{*}{$6.59(0.037)$} \\
\hline Amikacin $^{\mathrm{b}}$ & $0(0.00)$ & $12(40.00)$ & $18(60.00)$ & \\
\hline Chloramphenicol $^{\mathrm{a}}$ & $43(51.19)$ & $1(1.19)$ & 40 (47.62) & \multirow{2}{*}{$0.99(0.607)$} \\
\hline Chloramphenicol $^{\text {b }}$ & $13(43.33)$ & $0(0.00)$ & $17(56.67)$ & \\
\hline Doxycyclin $^{a}$ & $72(85.71)$ & $7(8.33)$ & $5(5.95)$ & \multirow{2}{*}{$1.81(0.404)$} \\
\hline Doxycycline ${ }^{b}$ & $3(10.00)$ & $23(76.67)$ & 4 (13.33) & \\
\hline Co-trimoxazole ${ }^{\mathrm{a}}$ & $54(64.29)$ & 12 (14.29) & $18(21.43)$ & \multirow{2}{*}{$0.54(0.762)$} \\
\hline Co-trimoxazole ${ }^{b}$ & $18(60.00)$ & $6(20.00)$ & $6(20.00)$ & \\
\hline
\end{tabular}

TABLE 3: Antimicrobial sensitivity pattern of Staphylococcus aureus strains to different antimicrobial agents with regard to MetS status

a: non-metabolic syndrome participants; b: metabolic syndrome participants

The resistances to three and more antibiotics (multidrug-resistant) were screened. Regarding metabolic syndrome status, $72.62 \%$ of isolates were multidrug-resistant in non-metabolic syndrome participants versus $63.33 \%$ in metabolic syndrome participants (Figure 4).

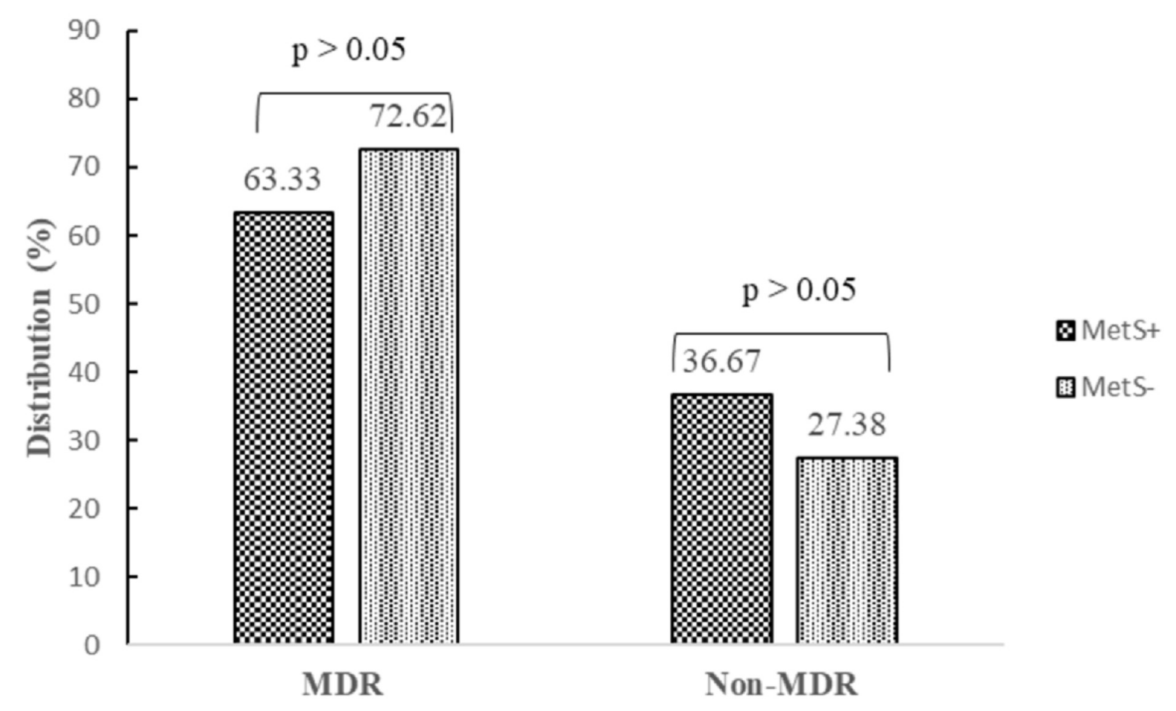

FIGURE 4: Multidrug-resistant nature of Staphylococcus aureus according to metabolic syndrome status 
Table 4 shows the multidrug-resistant nature of Staphylococcus aureus among participants with regards to gender and age. Among Staphylococcus aureus isolates, $74.60 \%$ were multidrug-resistant in females and $64.71 \%$ in males. The distribution of multidrug-resistance was significantly different in participants' age group ( $\mathrm{p}=0.0094$, chi-square=9.40). Higher Staphylococcus aureus multidrug-resistance was observed in the age group $\geqslant 70$ years $(100.00 \%)$, followed by the age group 60 to <70 (78.57\%) and 30 to <40 (77.78\%).

\begin{tabular}{|c|c|c|c|c|}
\hline \multicolumn{2}{|l|}{ Variables } & Non-MDR (N (\%)) & MDR (N (\%)) & $\mathrm{X}^{2}$ (probability) \\
\hline \multirow{2}{*}{ Sex } & Male & 18 (35.29) & $33(64.71)$ & \multirow{2}{*}{$1.32(0.250)$} \\
\hline & Female & $16(25.40)$ & 47 (74.60) & \\
\hline \multirow{6}{*}{ Age group (year) } & $20-<30$ & $12(38.71)$ & $19(61.29)$ & \multirow{6}{*}{$9.40(0.0094)$} \\
\hline & $30-<40$ & 2 (22.22) & 7 (77.78) & \\
\hline & $40-<50$ & $11(40.74)$ & $16(59.26)$ & \\
\hline & $50-<60$ & $6(31.58)$ & $13(68.42)$ & \\
\hline & $60-<70$ & $3(21.43)$ & $11(78.57)$ & \\
\hline & $\geq 70$ & $0(0.00)$ & $14(100.00)$ & \\
\hline
\end{tabular}

TABLE 4: Multidrug-resistant nature of Staphylococcus aureus among participants with regards to gender and age

MDR: multidrug-resistant; MetS: metabolic syndrome

\section{Discussion}

The frequency of MRSA in the gastrointestinal tract of metabolic syndrome patients in Mbouda AD LUCEM and District Hospitals was investigated. The prevalence of the mecA gene confirming methicillin-resistant Staphylococcus aureus was $79.82 \%(\mathrm{n}=91)$. The frequency of MRSA was higher in participants with metabolic syndrome (80.00\%) as compared to non-metabolic syndrome (79.76\%) participants without significant difference ( $\mathrm{p}=0.977)$. Results also indicated that the frequency of MRSA $(91 ; 79.83 \%)$ was higher than that of MSSA (23; 20.17\%). The frequency of MRSA in males was 41 (80.39\%) and that of females was 50 (79.37\%). A high frequency of MRSA was observed in participants aged 60 to $<70$ and $\geqslant 70$ years, that is, $92.86 \%$ and $100.00 \%$, respectively.

The human intestinal tract harbours a large number of cultivable bacteria such as Staphylococcus aureus. The colonization of the gastrointestinal tract by Staphylococcus aureus has been documented as potential sources of both endogenous and exogenous staphylococcal infections [5]. In community-acquired MRSA, Beilman et al. have reported that the prevalence rate of MRSA is up to $92 \%$. Kock et al. have reviewed 15 studies and revealed that $13 \%$ to $74 \%$ of worldwide staphylococcal infections are caused by MRSA [16]. MRSA colonizes body niches as any Staphylococcus aureus and the most prevalent site varies between studies as for MSSA.

The antimicrobial susceptibility test revealed Staphylococcus aureus isolates had a higher resistance to oxacillin in metabolic syndrome participants as compared to non-metabolic syndrome participants, that is, $100.00 \%$ and $92.86 \%$, respectively; non-significant at $\mathrm{p}=0.322$ (chi-square $=2.26$ ). The amikacin susceptibility profile was significantly different in metabolic syndrome and non-metabolic syndrome participants $(\mathrm{p}=0.037$, chi-square $=6.59)$. In metabolic syndrome participants, eight $(9.52 \%), 17(20.24 \%)$, and $59(70.24 \%)$ of Staphylococcus aureus were resistant,intermediate, and sensitive, respectively, to amikacin versus 0 (0.00\%), 12 (40.00\%), and 18 (60.00\%), respectively, in non-metabolic syndrome participants. In nonmetabolic syndrome participants, doxycycline had the highest overall resistance of $85.71 \%$, followed by cotrimoxazole (64.29\%) and Chloramphenicol (51.19\%) while amikacin had the highest overall sensitivity of $70.24 \%$, followed by gentamicin (64.29\%). These results show a marked increase in the resistance of Staphylococcus aureus isolates in metabolic syndrome as compared to non-metabolic syndrome participants.

Metabolic syndrome is a disorder of energy use and storage, characterized by central obesity, dyslipidemia, raised blood pressure and high blood sugar levels [17]. Disorders related to the metabolic syndrome could explain a high frequency of resistance of Staphylococcus aureus because it has been shown 
that Staphylococcus aureus pathogenesis seems to be closely linked to glucose availability in vitro and in humans [18]. Pomposelli et al. also demonstrated that hospitalized patients who are hyperglycaemic seem to be at a higher risk of Staphylococcus aureus infection [19].

Antibiotics multidrug-resistance defined as acquired non-susceptibility to at least one agent in three or more antimicrobial categories was studied. In females, $74.60 \%$ of Staphylococcus aureus isolates were multidrug-resistant, and in males, $64.71 \%$. Of isolates, $72.62 \%$ were multidrug-resistant in non-metabolic syndrome participants versus $63.33 \%$ in metabolic syndrome participants. Higher Staphylococcus aureus multidrug resistance was observed in the age group $\geqslant 70$ years $(100.00 \%)$ followed by the age group 60 to $<70(78.57 \%)$ and 30 to $<40(77.78 \%)$. The explanation for the observed variability may be linked to the differences in the sample size and the antibiotic prescription practices in the study area.

Although this study presents data from metabolic syndrome, patients where information on Staphylococcus aureus resistance is extremely limited, we have to acknowledge some limitations. The generalizability of the data might be compromised by sampling biases. In addition, the data should not be generalised to the entire country. We did not collect data in patients below 20 years. In spite of the highlighted limitations, it is still our position that this study presents vital information on the intestinal carriage of MRSA in metabolic syndrome patients. Therefore, the data has an important implication for the quality of patient care and infection control practices.

\section{Conclusions}

This study suggests that metabolic syndrome patients harbour MRSA strains in their intestines even as the difference was not statistically significant with non-metabolic syndrome participants. The need for appropriate antimicrobial use to halt, or at least limit, the spread of resistance is suggested in the care of metabolic syndrome patients and the entire population. The prevention of intestinal carriage of Staphylococcus aureus should be an important clinical concern.

\section{Additional Information \\ Disclosures}

Human subjects: Consent was obtained by all participants in this study. Cameroon National Ethics Committee (CNEC), Ministry of Public Health issued approval 2018/06/ 1054/CE/CNERSH. Participants received oral and written information about the study and signed an informed consent form. The protocol was approved by the Cameroon National Ethics Committee (CNEC), Ministry of Public Health. The study was conducted in agreement with the Declaration of Helsinki. Animal subjects: All authors have confirmed that this study did not involve animal subjects or tissue. Conflicts of interest: In compliance with the ICMJE uniform disclosure form, all authors declare the following: Payment/services info: All authors have declared that no financial support was received from any organization for the submitted work. Financial relationships: All authors have declared that they have no financial relationships at present or within the previous three years with any organizations that might have an interest in the submitted work. Other relationships: All authors have declared that there are no other relationships or activities that could appear to have influenced the submitted work.

\section{Acknowledgements}

The authors would like to thank all the staff of the Mbouda AD LUCEM and District Hospitals and the participants for their support towards the successful completion of the study. Authorship Statement: WTJM performed the sampling and data collection. WTJM and VK participated in the analysis of data. WTJM and VK drafted the manuscript. VK designed the study. All of the authors read the manuscript and approved the final version prior to submission.

\section{References}

1. Befus M, Lowy FD, Miko BA, Mukherjee D V, Herzig CTA, Larson EL: Obesity as a determinant of Staphylococcus aureus colonization among inmates in maximum-security prisons in New York State. Am J Epidemiol. 2015, 182:494-502. 10.1093/aje/kwv062

2. Biadgilign S, Mgutshini T, Haile D, Gebremichael B, Moges Y, Tilahun K: Epidemiology of obesity and overweight in sub-Saharan Africa: a protocol for a systematic review and meta-analysis. BMJ Open. 2017, 7:017666. 10.1136/bmjopen-2017-017666

3. Williams RE: Healthy carriage of Staphylococcus aureus: its prevalence and importance. Bacteriol Rev. 1963, 27:56-71.

4. Acton DS, Tempelmans Plat-Sinnige MJ, van Wamel W, de Groot N, van Belkum A: Intestinal carriage of Staphylococcus aureus: how does its frequency compare with that of nasal carriage and what is its clinical impact?. European Journal of Clinical Microbiology \& Infectious Diseases. 2009, 28:115. 10.1007/s10096008-0602-7

5. Sollid JUE, Furberg AS, Hanssen AM, Johannessen M: Staphylococcus aureus: determinants of human carriage. Infect Genet Evol. 2014, 21:531-541. 10.1016/J.MEEGID.2013.03.020

6. Rodriguez M, Hogan PG, Burnham C-AD, Fritz SA: Molecular epidemiology of Staphylococcus aureus in households of children with community-associated S aureus skin and soft tissue infections. J Pediatr. 2014, 
164:105-111. 10.1016/j.jpeds.2013.08.072

7. Kropec A, Huebner J, Riffel M, Bayer U, Benzing A, Geiger K, Daschner FD: Exogenous or endogenous reservoirs of nosocomial Pseudomonas aeruginosa and Staphylococcus aureus infections in a surgical intensive care unit. Intensive Care Med. 1993, 19:161-165. 10.1007/bf01720533

8. Lee SY, Kim JY, Kim JH, et al.: A case of primary infective endocarditis caused by community-associated methicillin-resistant Staphylococcus aureus in a healthy individual and colonization in the family. Yonsei Med J. 2009, 50:152-155. 10.3349/ymj.2009.50.1.152

9. Wielders CLC, Fluit AC, Brisse S, Verhoef J, Schmitz FJ: mecA gene is widely disseminated in Staphylococcus aureus population. J Clin Microbiol. 2002, 40:3970-3975. 10.1128/jcm.40.11.3970-3975.2002

10. Marbou WJT, Kuete V: Prevalence of metabolic syndrome and is components in Bamboutos Division's adults, West Region of Cameroon. Biomed Res Int. 2019, 2019:1-12. 10.1155/2019/9676984

11. Alberti KGMM, Eckel RH, Grundy SM, et al.: Harmonizing the metabolic syndrome. Circulation. 2009, 120:1640-1645. 10.1161/CIRCULATIONAHA.109.192644

12. Hassanzadeh S, Pourmand MR, Afshar D, Dehbashi S, Mashhadi R: TENT: a rapid DNA extraction method of Staphylococcus aureus. Iran J Public Health. 2016, 45:1093-1095.

13. Brakstad OG, Aasbakk K, Maeland JA: Detection of Staphylococcus aureus by polymerase chain reaction amplification of the nuc Gene. J Clin Microbiol. 1992, 30:1654-1660.

14. CLSI. M100-S10. Performance standard for antimicrobial susceptibility testing . (2017). https://clsi.org/media/2663/m100ed29_sample.pdf.

15. Kumurya A: One step PCR for detection of Staphylococcus aureus specific sequence gene and mecA gene in Northwestern Nigerian hospitals. Antimicrob Resist Infect Control. 2015, 4:73-79. 10.1186/2047-2994-4-s1p196

16. Köck R, Becker K, Cookson B, et al.: Methicillin-resistant Staphylococcus aureus (MRSA): burden of disease and control challenges in Europe. Euro Surveill. 2010, 15:19688.

17. Cornier M-A, Dabelea D, Hernandez TL, et al.: The metabolic syndrome. Endocr Rev. 2008, 29:777-822. 10.1210/er.2008-0024

18. Vitko NP, Grosser MR, Khatri D, Lance TR, Richardson AR: Expanded glucose import capability affords Staphylococcus aureus optimized glycolytic flux during infection. MBio. 2016, 7:e00296. 10.1128/MBIO.00296-16

19. Pomposelli JJ, Baxter JK, Babineau TJ, et al.: Early postoperative glucose control predicts nosocomial infection rate in diabetic patients. J Parenter Enter Nutr. 1998, 22:77-81. 10.1177/014860719802200277 